biomarker severity from $\mathrm{CON}$ to $\mathrm{AD}$, with $\mathrm{SCD}$ showing a small, but significant difference from CON in the EC and HPC. Results: Qualitatively, morphometry results showed a gradient of changes from $\mathrm{CON}$ to $\mathrm{AD}$ in the EC and HPC (Figure 1), especially for the left EC and right HPC, but with SCD not significantly different from CON. Furthermore, compared to CON, SCD had significant lower right lingual surface $(p=.021)$, left posterior cingulate thickness $(p=.026)$, right rostral anterior cingulate thickness $(p=.012)$, left supramarginal surface $(p=.019)$ and volume $(p=.039)$, however none of those differences resisted FDR correction. Grading results (Figure 2) were similar to morphometry, with a gradient of changes from $\mathrm{CON}$ to $\mathrm{AD}$, especially for the left $\mathrm{EC}$ and right HPC, but no significant difference between SCD and CON. Conclusions: Based on these results, baseline morphometric measures and TPMD grading scores for EC and HPC do not capture the difference related to the cognitive complaint between SCD and CON. Longitudinal follow-up is required to determine their positive predictive values for AD pathology.

\section{P3-375 CHARACTERISTIC BRAIN VOLUMETRIC CHANGES IN THE AD PRECLINICAL SIGNATURE}

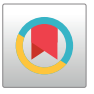

Paula Marcela Petrone ${ }^{1}$, Adriá Casamitjana ${ }^{2}$, Carles Falcon ${ }^{1}$, Miquel Artigues ${ }^{2}$, Grégory Operto ${ }^{1}$, Stavros Skouras ${ }^{1}$,

Raffaele Cacciaglia ${ }^{1}$, José Luis Molinuevo ${ }^{1}$, Verónica Vilaplana ${ }^{2}$, Juan Domingo Gispert ${ }^{1}$, Gemma Salvadó ${ }^{1}$ and the Alzheimer's Disease Neuroimaging Initiative, ${ }^{1}$ Barcelonaßeta Brain Research Center, Barcelona, Spain; ${ }^{2}$ Universitat Politècnica de Catalunya, Barcelona, Spain.Contact e-mail: petronepau@gmail.com

Background:In the last decade, magnetic resonance imaging has unveiled specific $\mathrm{AD}$ alterations at different stages of the $\mathrm{AD}$ pathophysiologic continuum that conform what has been established as the AD signature. To which extent MRI can detect structural changes at the preclinical asymptomatic stage of AD -the preclinical AD signature- is still an area open for exploration. Methods: Longitudinal Magnetic Resonance Imaging (MRI-T13D) data was acquired from a subset of the ADNI cohort comprising 817 subjects (174 controls, 125 preclinical, $518 \mathrm{MCI} /$ Dementia due to $\mathrm{AD}$, at baseline) for which cerebrospinal fluid (CSF) biomarker data is publicly available. For each subject, volumetric changes in brain structure between two consecutive acquisition timepoints (average time interval of 2 years) were defined by calculating jacobian determinants using the SPM longitudinal Voxel-Based Morphometry pipeline. Statistical analysis was performed to identify brain regions with significantly different changes between five progressive groups: (1) Normal controls, (2) Normal control subjects that convert to amyloid positive, (3) Preclinical subjects, (4) Preclinical subjects that become symptomatic, (5) Symptomatic (MCI and AD) subjects. Results: We define the preclinical $A D$ signature as statistically significant structural brain changes between normal controls vs. preclinical subjects (group 1 vs 3). We found certain brain regions that show early subtle atrophy (e.g. Middle Temporal). Other regions show volume increments (e.g. Hippocampus) whereas they display longitudinal atrophy in symptomatic stages (Figure 1), probably resulting in expansion of CSF. Thus, the characterized preclinical AD signature presents regions that overlap with the well-established AD signature (e.g. Precuneus), and others that do not (e.g. Caudate). We will also describe changes that are specific for groups (2) and (4). Conclusions: Our work supports the idea that there are brain volumetric changes specific to preclinical $\mathrm{AD}$ subjects and defines the preclinical $\mathrm{AD}$

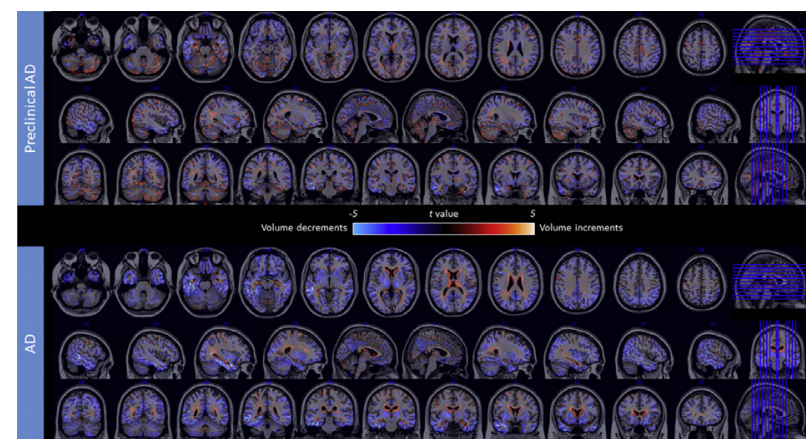

Figure 1. T-maps of the longitudinal Jacobian determinant differences between (i) normal controls and preclinical AD groups (top) and (ii) normal controls and $\mathrm{AD}$ groups (bottom). Colormap indicates volume contractions (blue) and expansions (red). Apparent volume increments can be observed mostly in periventricular regions, likely resulting in the expansion of the CSF.
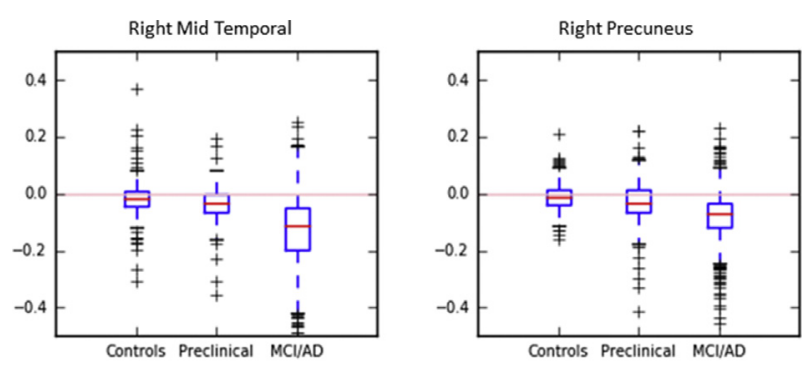

Left Hippocampus
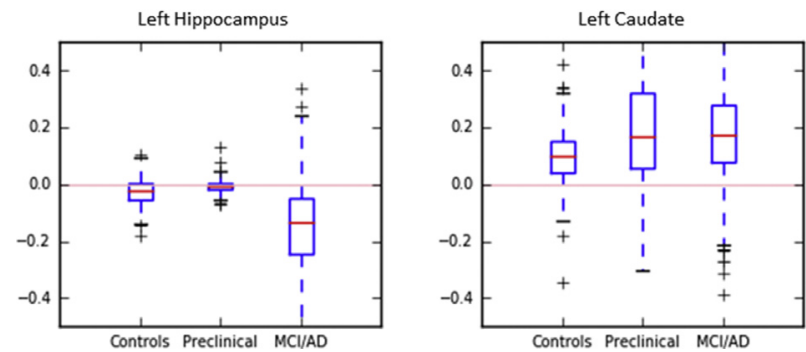

Figure 2. Distribution of jacobian determinant values for each population group (i) normal controls, (ii) preclinical subjects, (iii) MCI/AD, according to brain region of Interest (ROIs). Average over significant voxels for each jacobian determinant is provided. Positive values indicate volume expansions and negative values indicate contractions. Units of volume change are arbitrary but consistent throughout the ROIs.

signature based on longitudinal data. While some regions show a pattern of atrophy that overlaps with the AD signature, other specific regions exhibit changes that are unique to this early asymptomatic AD stage. Promising applications of these jacobian determinant features may, in the future, be used for subject classification.

\section{P3-376 CEREBRAL MICROINFARCT INFLUENCES STRUCTURAL NETWORK TOPOLOGY IN ALZHEIMER'S DISEASE AND COGNITIVE IMPAIRMENT NO DEMENTIA}

Liwen Zhang ${ }^{1,2}$, Geert Jan Biessels ${ }^{3}$, Hee Youn Shim ${ }^{1}$, Yng Miin ${ }^{1}$, Saima Hilal $^{4}$, Narayanaswamy Venketasubramanian ${ }^{5}$, Boon Yeow Tan ${ }^{6}$, Christopher Chen ${ }^{2}$, Juan Zhou ${ }^{1},{ }^{1}$ Duke-National University of Singapore Medical School, Singapore, Singapore; ${ }^{2}$ Yong Loo Lin School of Medicine, 